\title{
Effects of High White and Brown Sugar Consumption on Serum Level of Brain-Derived Neurotrophic Factor, Insulin Resistance, and Body Weight in Albino Rats
}

\author{
Ali Shamsi-Goushki ', Zinat Mortazavi ${ }^{2}$, Mohammad Ali Mirshekar ${ }^{3,4, *}$, Fatemeh Behrasi ${ }^{1}$, Nasroallah Moradi-Kor ${ }^{5}$, \\ Rasul Taghvaeefar ${ }^{1}$ \\ 'Student Research Committee, ${ }^{2}$ Health Promotion Research Center, ${ }^{3}$ Clinical Immunology Research Center, and, ${ }^{4}$ Department of Physiology, School of Medicine, \\ Zahedan University of Medical Sciences, Zahedan; ${ }^{5}$ Research Center of Physiology, Semnan University of Medical Sciences, Semnan, Iran
}

Background: In recent decades, consumption of simple sugars has increased dramatically, which contributes to health problems including insulin resistance and obesity. In this study, we investigated the effects of high concentrations of white sugar (WS) and brown sugar (BS) on serum concentration of brain-derived neurotrophic factor (BDNF), insulin resistance, and body weight in albino rats.

Methods: Twenty-four male Wistar rats were randomly divided into three groups: control, a group treated with $15 \%$ WS, and a group treated with $15 \%$ BS. Rats were given WS and BS by gavage (daily) for 42 days. At the end of the intervention period, serum level of BDNF, insulin resistance, and body weight were measured.

Results: Body weight and insulin resistance were significantly increased and serum BDNF level was decreased in both WS and BS groups compared to the control group $(P<0.05)$. In the WS-treated rats, the amount of changes in the insulin resistance, body weight, and serum BDNF level was greater compared to that in BS-treated ( $P<$ 0.05).

Conclusion: Due to the adverse effects of consuming high levels of WS and BS on serum level of BDNF, insulin resistance, and body weight, high intakes of these sweeteners are not recommended.

Key words: Brown sugar, White sugar, Brain-derived neurotrophic factor, Insulin resistance
Received April 27, 2020

Reviewed June 8, 2020

Accepted September 21, 2020

${ }^{*}$ Corresponding author

Mohammad Ali Mirshekar

(iD https://orcid.org/0000-0002-0449-6440

Clinical Immunology Research Center, Zahedan University of Medical Sciences, Persian Gulf Highway, Zahedan 009816875569, Iran Tel: +98-54-3329-5720

Fax: +98-54-3329-5720

E-mail:ma_mib78@yahoo.com

\section{INTRODUCTION}

In recent decades, consumption of sugars has increased rapidly, with negative effects on human health. ${ }^{1}$ Excessive consumption of sweetened beverages causes metabolic diseases such as type 2 diabetes mellitus (T2DM), obesity, and cardiovascular diseases. ${ }^{2}$ The World Health Organization (WHO) recommends that the daily consumption of sugars not be greater than $5 \%-10 \%$ of the overall daily caloric intake. ${ }^{3}$ Problems associated with high consumption of simple sugars are obesity and, consequently, insulin resistance. ${ }^{2}$ Insulin resistance has on inverse effect on serum level of brain-derived neurotrophic factor (BDNF). ${ }^{4}$
BDNF is one of the factors of the neurotrophin family that is widely secreted in some areas of mammalian cerebral tissue, such as the hippocampal region. ${ }^{5}$ BDNF plays a vital role in development and maintenance of central and peripheral nervous system health and synaptic plasticity. Serum level of BDNF has been reported to decrease in patients with insulin resistance and diabetes. ${ }^{4}$ A common belief exists that brown sugar (BS) is less damaging to health than white sugar (WS), but preliminary experimental information on this matter is scarce. The purpose of this study was to investigate the effects of high WS and BS consumption on serum level of BDNF, insulin resistance, and body weight in albino rats. 


\section{METHODS}

In this experimental study, 24 healthy male Wistar rats, aged 3 months and weighing 200-250 g, were purchased from the laboratory of the animal research center of Zahedan University of Medical Sciences. All stages of the study were designed and implemented according to the guidelines of the laboratory animal ethics committee of Zahedan University of Medical Sciences (No. IR.ZAUMS.REC.1398.448). The rats were exposed to dark/light cycles and allowed free access to diet and water. The environmental temperature was kept at $24^{\circ} \mathrm{C} \pm 2^{\circ} \mathrm{C}$ with $60 \% \pm 5 \%$ humidity. The intervention was started after 2 weeks of acclimatization. ${ }^{6}$

\section{Solution preparation}

The percentages of sugar solutions were calculated based on the daily caloric intake of rats (15-16 g). According to the WHO recommendation that healthy people should not consume more than $5 \%$ of their daily calories as sugar, $15 \%$ of the daily caloric intake of rats was determined to be $2.25 \mathrm{~g}$. For each of the sugar groups, the concentration of total sugar in solution was $2.25 \mathrm{~g} \mathrm{wt}$ : vol (comparable to sugar-sweetened beverages [SSBs] typically consumed by humans) in distilled water.

\section{Experimental design and dietary regimen}

The rats were divided into three equal groups (eight rats per each group) including: ${ }^{7}$ group 1, control group; group 2: group treated with $15 \%$ of WS; group 3, group treated with $15 \%$ of BS. Rats had free access to water and food. The control group received normal saline, and the intervention groups received WS or BS (Haft Tapeh Sugar Cane Company, Ahvaz, Iran) by oral gavage (daily) for 42 days. ${ }^{8}$

\section{Collecting samples}

At the end of the experimental period, the rats were anesthetized with $60 \mathrm{mg} / \mathrm{kg}$ of ketamine and $5 \mathrm{mg} / \mathrm{kg}$ of xylazine (Alfasan, Woerden, the Netherlands) after 12 hours of overnight fasting. ${ }^{6}$ Blood samples were collected through cardiac puncture for determination of biochemical parameters. Blood samples were centrifuged at $3,000 \mathrm{rpm}$ for 10 minutes to obtain serum. The serum samples were isolated in special micro-tubes and frozen at $-75^{\circ} \mathrm{C}$ until biochemi- cal assessments. ${ }^{6}$ At the beginning and end of the intervention, body weight was measured using a digital scale (EK610i; A\&D, Tokyo, Japan). At the end of the intervention, the rats were anesthetized with an intraperitoneal injection of a mixture of ketamine/xylazine for euthanasia.

\section{Plasma biochemical assays}

Serum levels of insulin and BDNF (Bioassay Technology Laboratory, Shanghai, China) were measured using rat-specific kits by enzyme-linked immunosorbent assay (ELISA) using an ELISA reader (Stat Fax 2100; Awareness Technology, Palm City, FL, USA).

\section{Estimation of insulin resistance}

The homeostatic model assessment for insulin resistance (HOMA-IR) formula was used to estimate insulin resistance. ${ }^{10}$

\section{Statistical analysis}

Statistical analysis was performed using GraphPad Prism version 8.0 (GraphPad, San Diego, CA, USA). The Kolmogorov-Smirnov test was utilized to test data required for various assessments of normal distribution. If the distribution was normal, data were presented as mean \pm standard error of the mean. HOMA-IR and BDNF were analyzed at the end of the intervention using one-way analysis of variance (ANOVA) after applying Tukey's post hoc test. Body weight data were analyzed by repeated one-way ANOVA followed by Tukey's post-hoc test. The $P$-values $<0.05$ were considered significant.

\section{RESULTS}

\section{Effects of WS and BS consumption on rat body weight}

The effects of WS and BS consumption on body weight were measured before and after of the intervention. Excessive consumption of WS and BS significantly increased body weight in the intervention groups compared to the control group at the end of the intervention $(P<0.05)$. Interestingly, WS caused greater increase in body weight than BS, and this difference was significant $(P<0.05)$. These differences were not significant before the intervention. 


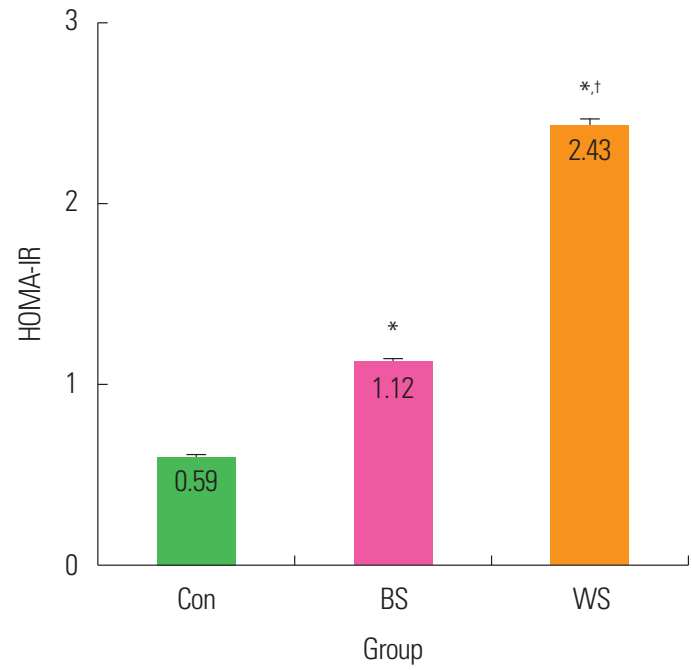

Figure 1. Effects of white and brown sugar consumption on insulin resistance in rats. Values are presented as mean \pm standard deviation $(n=8)$ and analyzed by one-way analysis of variance and Tukey's post-hoc tests. ${ }^{*} P<0.001$ compared to the Con group; ${ }^{\dagger} P<0.001$ compared to the BS group. HOMA-IR, homeostatic model assessment for insulin resistance; Con, control; BS, brown sugar; WS, white sugar.

\section{Effects of WS and BS consumption on insulin resistance in rats}

The effects of WS and BS consumption on HOMA-IR were measured at the end of the intervention and are shown in Fig. 1. HOMA-IR values were significantly increased in both WS and BS compared with the control group $(P<0.05)$.

\section{Effects of WS and BS consumption on serum level of BDNF in the rats}

The effects of WS and BS consumption on serum level of BDNF were measured at the end of the intervention and are shown in Fig. 2. The serum level of BDNF in WS and BS groups was significantly decreased compared to that of the control group $(P<0.05)$, and mean BDNF concentration in the WS group was less than that in the $\mathrm{BS}$ group $(P<0.05)$.

\section{DISCUSSION}

In our study, the effects of high consumption of WS and BS on serum level of BDNF, insulin resistance, and body weight in Wistar rats were investigated. We found that body weight and HOMA-IR were significantly increased in the WS and BS groups in comparison with the control group. Zhao and Castonguay ${ }^{11}$ reported that

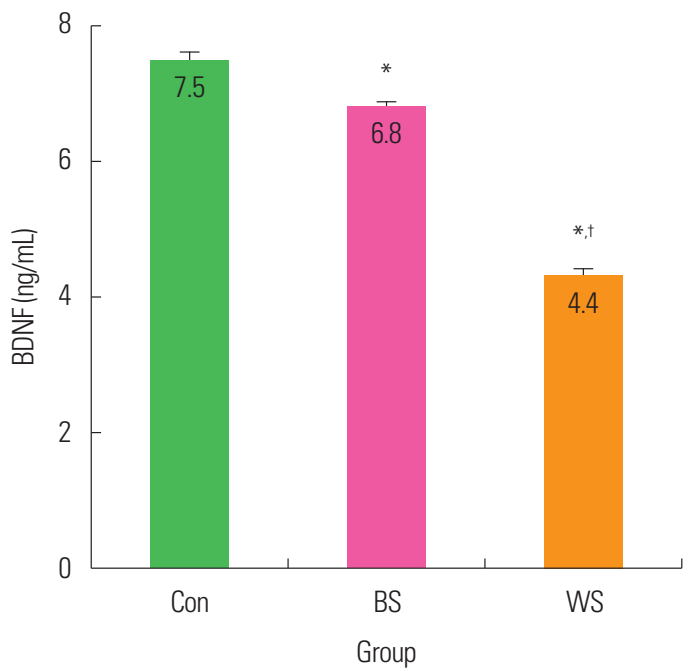

Figure 2. Effects of white and brown sugar consumption on serum level of BDNF in rats Values are presented as mean \pm standard deviation $(n=8)$ and analyzed by one-way analysis of variance and Tukey's post-hoc tests. ${ }^{*} P<0.001$ compared to the Con group; ${ }^{\dagger} P<0.001$ compared to the BS group. BDNF, brain-derived neurotrophic factor; Con, control; BS, brown sugar; WS, white sugar.

long-term consumption of sugar can increase the risk of obesity and other associated diseases. Lozano et al. ${ }^{12}$ investigated whether a high fructose and high-fat diet can increase the risk of diabetes and its complications and showed that 2 months of consumption of a high fructose and high-fat diet can cause increases in body weight and HOMA-IR. Gómez-Crisóstomo et al. ${ }^{8}$ conducted a study to investigate the effects of high-calorie diets on insulin sensitivity in rats and showed that 1-week consumption of solution containing $30 \%$ sugar had little effect on insulin sensitivity. These results indicate that development of insulin resistance depends on the amount of sugar in solution and the duration of the intervention. The harmful effects of SSBs may be explained by de novo lipogenesis, resulting in weight gain and fat accumulation in the abdomen. ${ }^{13}$ Consumption of SSBs may also aggravate inflammation and insulin resistance by altering expression of related genes, such as interleukin- 6 and monocyte chemoattractant protein- $1 .^{14}$

In our experimental study, we found that serum level of BDNF in WS and BS groups was significantly decreased compared to that in the control group. The WS group exhibited greater weight gain compared to the BS group, explaining the differences in HOMAIR and BDNF between WS and BS. To explain how diet may impact neural plasticity, studies have shown that obesity reduces the concentration and function of BDNF, and that high sugar diets can 
enhance the production of free radicals in the liver and brain. Neurotrophic factors such as BDNF protect neurons against oxidative stress, and the high-fat sucrose diet can attenuate neurotransmitter secretion that is typically modulated by BDNF and synapsin $1 .{ }^{4} \mathrm{In}-$ sulin resistance reduces the expression of BDNF in sensory and motor roots. ${ }^{15}$ BDNF concentration is also decreased in the plasma of T2DM patients. Therefore, there is an inverse correlation between BDNF and fasting plasma glucose/HOMA-IR index. ${ }^{16}$

Chromium is an element that plays a role in improving insulin sensitivity. There is three times as much of this element in BS as in WS. ${ }^{8}$ Therefore, WS is more effective than BS for increasing HOMAIR and decreasing serum level of BDNF. ${ }^{8}$ In this context, Feng et al. ${ }^{17}$ reported that chromium malate improved glucose metabolism in diabetic rats. Another reason that BS more effectively lowers HOMA-IR than does WS is due to its high antioxidant values. ${ }^{8}$

Finally, we conclude that excessive consumption of WS and BS has negative effects on serum level of BDNF, insulin resistance, and body weight. The negative effects of BS on these factors are less than those of WS. We also recommend that future research examine the effects of different doses of WS and BS intake on biochemical factors in healthy and diabetic albino rats. Histology phenotypes (liver, adipose, muscle, brain) and immunohistochemistry were not identified in this study.

\section{CONFLICTS OF INTEREST}

The authors declare no conflict of interest.

\section{ACKNOWLEDGMENTS}

The authors acknowledge the Research Affairs of the Health Promotion Research Center, Deputy of Research and Technology (Grant no. 9481) Zahedan University of Medical Sciences for providing facilities and financial support.

\section{AUTHOR CONTRIBUTIONS}

Study concept and design: MAM; acquisition of data: ASG; analysis and interpretation of data: ZM; drafting of the manuscript: ASG, MAM, NMK, and FB; critical revision of the manuscript:
NMK; statistical analysis: RT; obtained funding: ASG; administrative, technical, or material support: MAM; and study supervision: MAM.

\section{REFERENCES}

1. Hsiao A, Wang YC. Reducing sugar-sweetened beverage consumption: evidence, policies, and economics. Curr Obes Rep 2013;2:191-9.

2. Prinz P. The role of dietary sugars in health: molecular composition or just calories? Eur J Clin Nutr 2019;73:1216-23.

3. Yang Q, Zhang Z, Gregg EW, Flanders WD, Merritt R, Hu FB. Added sugar intake and cardiovascular diseases mortality among US adults. JAMA Intern Med 2014;174:516-24.

4. Rozanska O, Uruska A, Zozulinska-Ziolkiewicz D. Brain-derived neurotrophic factor and diabetes. Int J Mol Sci 2020;21: 841.

5. Bathina S, Das UN. Brain-derived neurotrophic factor and its clinical implications. Arch Med Sci 2015;11:1164-78.

6. Ganugula R, Arora M, Jaisamut P, Wiwattanapatapee R, Jørgensen HG, Venkatpurwar VP, et al. Nano-curcumin safely prevents streptozotocin-induced inflammation and apoptosis in pancreatic beta cells for effective management of Type 1 diabetes mellitus. Br J Pharmacol 2017;174:2074-84.

7. Molteni R, Barnard RJ, Ying Z, Roberts CK, Gómez-Pinilla F. A high-fat, refined sugar diet reduces hippocampal brain-derived neurotrophic factor, neuronal plasticity, and learning. Neuroscience 2002;112:803-14.

8. Gómez-Crisóstomo NP, de la Cruz AD, Camacho LJU, de la Cruz-Hernández EN, Martínez-Abundis E. Chronic consumption of refined but not brown sugar induces Hypertriglyceridemia and fat accumulation in rat liver. Acad J Sci Res 2017;5: 451-9.

9. Ranjbar Kohan N, Nazifi S, Tabandeh MR, Ansari Lari M. Effect of L-carnitine supplementation on Apelin and Apelin Receptor (Apj) expression in cardiac muscle of obese diabetic rats. Cell J 2018;20:427-34.

10. Oza MJ, Kulkarni YA. Formononetin treatment in type 2 diabetic rats reduces insulin resistance and hyperglycemia. Front Pharmacol 2018;9:739. 
11. Zhao C, Castonguay TW. Effects of free access to sugar solutions on the control of energy intake. Food Rev Int 2017;33: 105-22.

12. Lozano I, Van der Werf R, Bietiger W, Seyfritz E, Peronet C, Pinget $\mathrm{M}$, et al. High-fructose and high-fat diet-induced disorders in rats: impact on diabetes risk, hepatic and vascular complications. Nutr Metab (Lond) 2016;13:15.

13. Lee G, Han JH, Maeng HJ, Lim S. Three-month daily consumption of sugar-sweetened beverages affects the liver, adipose tissue, and glucose metabolism. J Obes Metab Syndr 2020;29: 26-38.

14. Kanda H, Tateya S, Tamori Y, Kotani K, Hiasa K, Kitazawa R, et al. MCP-1 contributes to macrophage infiltration into adi- pose tissue, insulin resistance, and hepatic steatosis in obesity. J Clin Invest 2006;116:1494-505.

15. Eslami R, Gharakhanlou R, Kazemi A, Dakhili AB, Sorkhkamanzadeh G, Sheikhy A. Does endurance training compensate for neurotrophin deficiency following diabetic neuropathy? Iran Red Crescent Med J 2016;18:e37757.

16. Krabbe KS, Nielsen AR, Krogh-Madsen R, Plomgaard P, Rasmussen P, Erikstrup C, et al. Brain-derived neurotrophic factor (BDNF) and type 2 diabetes. Diabetologia 2007;50:431-8.

17. Feng W, Zhao T, Mao G, Wang W, Feng Y, Li F, et al. Type 2 diabetic rats on diet supplemented with chromium malate show improved glycometabolism, glycometabolism-related enzyme levels and lipid metabolism. PLoS One 2015;10:e0125952. 